\title{
Quality of life in childhood epilepsy with lateralized epileptogenic foci
}

\author{
Krystyna A Mathiak ${ }^{1,2,3^{*}}$, Małgorzata Łuba², Klaus Mathiak ${ }^{3,4}$, Katarzyna Karzel ${ }^{2}$ Tomasz Wolańczyk \\ Elżbieta Szczepanik ${ }^{6}$, Paweł Ostaszewski ${ }^{2}$
}

\begin{abstract}
Background: Measuring quality of life (QOL) helps to delineate mechanisms underlying the interaction of disease and psychosocial factors. In adults, epileptic foci in the left temporal lobe led to lower QOL and higher depression and anxiety as compared to the right-sided foci. No study addressed the development of QOL disturbances depending on the lateralization of epileptogenic focus. The objective of our study was to examine QOL in children with lateralized epileptiform discharges.
\end{abstract}

Methods: Thirty-one parents of children with epilepsy filled the Health-Related Quality of Life in Childhood Epilepsy Questionnaire (QOLCE). Fifteen children had foci in the left hemisphere and sixteen in the right, as verified with Electroencephalography (EEG) examinations.

Results: We found a significant correlation between foci lateralization and reduced QOL (Spearman's rho $=0.361$, $\mathrm{p}<$ 0.046). Children with right hemispheric foci exhibited lower overall QOL, particularly in five areas: anxiety, social-activities, stigma, general-health, and quality-of-life.

Conclusions: We demonstrated for the first time that in children left- and right-hemispheric foci were associated with discordant QOL scores. Unlike in adults, foci in the right hemisphere led to worse emotional and social functioning demonstrating that seizures impact the brain differentially during development.

\section{Background}

Quality of life (QOL) is an important outcome measure in clinical research, taking into account patient's subjective evaluation of his own being and supplementing the traditional medical approach, based on the examination of symptoms, signs, and laboratory findings as defined from the doctor's perspective [1]. World Health Organization (WHO) defined QOL as 'individuals' perception of their position in life in the context of the culture and value systems in which they live and in relation to their goals, expectations, standards and concerns' [2]. The term incorporates multidimensional aspects of individuals' physical health, psychological state and social relationships. Quality of life is an important measure in epilepsy, which is often a chronic and debilitating condition and unique among the chronic illnesses due to the

\footnotetext{
* Correspondence: kamathiak@ukaachen.de

'Department of Child and Adolescent Psychiatry and Psychotherapy, RWTH Aachen University, Germany

Full list of author information is available at the end of the article
}

multidimensional impact on psychosocial functioning [3].

Childhood epilepsy is among the most prevalent and important neurological conditions in the developing years, affecting 3.6 to 4.2 per 1000 children in developed countries, and approximately double these rates in developing countries (for a review, see [4]). The consequences of epilepsy often evolve in a complex fashion and can be accounted for by a number of factors including the effects of brief but chronic dysfunctional episodes on the developing brain, the presence of comorbid disabilities, the subsequent cognitive problems, and the reaction of the individuals and their families to the diagnosis and treatment [5]. The improvement of clinical parameters only, even including remission, does not need to lead to the improvement of social functioning and subjective QOL [6,7].

The unique feature of epilepsy, among other chronic conditions, is its direct effect on the brain. This might contribute to more disturbed psychosocial functioning in patients diagnosed with epilepsy as compared to 
other chronic, stigmatizing disorders. Therefore our understanding of psychological and social effects of epilepsy can be enhanced by neuropsychological insights into the brain-behavior relationship [8]. Studying behavioral consequences of lateralized potentially epileptogenic foci may offer an insight into understanding these links.

Lateralized seizures have differential impact on behavior. It was observed that right temporal lobe seizures evoke experiential phenomena more often, including an intense sense of the familiar and unfamiliar, fear, and complex multisensory hallucinations [9]. Left-sided foci may lead to ictal outbursts of laughing (gelastic epilepsy) [10]. Depression and anxiety were typically observed in patients with left- versus right-sided temporal lobe epilepsy $[11,12]$. Andelman et al. [13] demonstrated that left temporal epilepsy is associated with higher level of anxious personality trait and a decreased self-assessment of QOL as compared to right-sided focus. A number of studies have demonstrated that lateralization patterns may change over the developmental period (for a review, see [14]). Upton and Thompson [15] showed that frontal epileptic focus has differential effect on cognitive functions in children, depending on age when the disease started. Their finding demonstrates that lateralized seizures impact behavior differently depending on age.

Although early epidemiological studies suggest that left temporal lobe epilepsy in children is related to increased behavioral problems, in particular hyperactivity, antisocial behavior and aggression, this finding has not been confirmed so far (for a review, see [16]). Cohen et al. [17] found disturbed prosody recognition in children with right-sided focus only, therefore demonstrating that in the developmental age lateralized seizures can influence neuropsychology differentially. Mathiak et al. [18], using generic scales, demonstrated higher level of disruptive behavior in children with right sided foci and more internal locus of control in children with left-hemispheric seizures. To assess complex multidimensional consequences of epilepsy, a broad assessment of QOL was recommended [19]. Studies of QOL in unilateral epileptic seizures have concentrated exclusively on adults.

The Health-Related Quality of Life in Childhood Epilepsy Questionnaire (QOLCE; [20]) examines QOL in a wide range of life domains including physical, cognitive, emotional, and social functioning and was developed and validated specifically for children with epilepsy. Such scales were shown to have higher sensitivity to disturbances in epilepsy than generic instruments [21]. Our objective was to assess whether there was an association between lateralized epileptiform discharges in childhood epilepsy and QOL. Children with right and left epileptiform discharges completed QOLCE scale. We hypothesized that foci lateralization correlates with a reduced QOL in children with unilateral epilepsy thus confirming a direct brain-behavior interaction influencing QOL during development.

\section{Methods \\ Subjects}

A group of 51 children from the Institute of Mother and Child in Warsaw and the Warsaw Hospital for Children was recruited based on hospital discharge cards. Patients were included in the presence of (1) unilateral epileptic focus as determined by presence of epileptiform activity in EEG examinations, (2) age in the range of 6-15 years, (3) normal intelligence (IQ above 85 in Wechsler's scale), and (4) no accompanying chronic diseases that was not a direct outcome of epilepsy, such as asthma or diabetes, or any disease that started before the onset of epileptic seizures. We included all the children meeting the inclusion criteria who visited the outpatient clinic during the time of the study and over $90 \%$ of them agreed to participate. The exclusion criteria were: (1) generalized discharges in an EEG or unclear lateralization; changing lateralization patterns in consecutive EEG examinations, (2) first epileptiform discharges which had occurred less than one year prior to the examination, or (3) anatomical malformations detected by cranial computed tomography or magnetic resonance. Twenty patients were excluded: 11 subjects had bilateral or generalized discharges; in four the disease was diagnosed less then one year earlier, and five had anatomical findings. Finally a group of 31 children (12 girls and 19 boys, mean age11.2 $\pm 2.7,16$ with right and 15 with the left hemispheric focus) took part in the study. Patient characteristics are summarized in Table 1. Prior to the study, a written informed consent was obtained from a caregiver as well as an oral consent from the child. The Ethics Committee of the Institute for Mother and Child in Warsaw, Poland approved the study protocol.

\section{Psychological measures}

Parents of children completed the Health-Related Quality of Life in Childhood Epilepsy Questionnaire (QOLCE; [20]; Polish version [22]), a reliable epilepsyspecific parental questionnaire for children aged 4-18 years. The original scale was validated in Australia as well as in USA and its high reliability (Cronbach's alpha 0.93 and 0.92 accordingly) as well as good validity were confirmed $[20,23]$. The Polish version was adapted in a group of 87 children with epilepsy aged 4-18 years and it retain psychometric values of the original, with very high reliability (Cronbach's alpha 0.97 ) as well as good validity [22]. The scale contains 91 items, measuring an overall QOL, with two generic single-item subscales (general health item and QOL item) and 14 subscales, 
Table 1 Individual clinical characteristics

\begin{tabular}{|c|c|c|c|c|c|c|c|}
\hline $\begin{array}{c}\text { Age } \\
\text { (years) }\end{array}$ & Sex & $\begin{array}{l}\text { Disease } \\
\text { (years) }\end{array}$ & $\begin{array}{l}\text { Medication } \\
\text { (years) }\end{array}$ & \multicolumn{2}{|c|}{$\begin{array}{l}\text { Antiepileptic drugs } \\
\text { at the time of } \\
\text { examination* }\end{array}$} & $\begin{array}{l}\text { Hospitalizations } \\
\text { per year }\end{array}$ & $\begin{array}{c}\text { Number of seizures } \\
\text { per month }\end{array}$ \\
\hline \multicolumn{8}{|c|}{ Left-hemispheric seizure focus } \\
\hline 7 & Boy & 1 & 1 & 1 & C & 1.00 & $<1$ \\
\hline 8 & Boy & 1 & 1 & 3 & $C \vee L$ & 2.00 & $1-9$ \\
\hline 8 & Boy & 5 & 5 & 2 & $C L$ & 0.50 & $1-9$ \\
\hline 9.5 & Girl & 5 & 3 & 1 & C & 0.20 & $1-9$ \\
\hline 10.5 & Boy & 2 & 1 & 1 & $\mathrm{O}$ & 1.00 & $1-9$ \\
\hline 11 & Boy & 4 & 4 & 3 & $\mathrm{VTCl}$ & 1.00 & $1-9$ \\
\hline 11 & Boy & 6 & 5 & 2 & $C L$ & 0.50 & $1-9$ \\
\hline 11.5 & Boy & 3 & 3 & 1 & $L$ & 0.50 & $>30$ \\
\hline 12 & Girl & 10 & 8 & 1 & $\mathrm{O}$ & 0.25 & $>30$ \\
\hline 12 & Girl & 8 & 2 & 1 & O & 0.18 & $>30$ \\
\hline 13 & Girl & 3 & 3 & 1 & $\mathrm{O}$ & 0.50 & $1-9$ \\
\hline 13 & Girl & 10 & 9 & 3 & $V L T$ & 0.67 & $<1$ \\
\hline 13 & Boy & 3 & 2 & 1 & $\mathrm{O}$ & 0.20 & $>30$ \\
\hline 13.5 & Girl & 5 & 5 & 1 & V & 0.80 & $<1$ \\
\hline 14.5 & Boy & 3 & 0 & 1 & V & 0.00 & $1-9$ \\
\hline 15 & Boy & 4 & 4 & 1 & O & 0.33 & $1-9$ \\
\hline \multicolumn{8}{|c|}{ Right-hemispheric seizure focus } \\
\hline 6 & Girl & 3 & 2 & 2 & $C V$ & 0.33 & $<1$ \\
\hline 6 & Boy & 3 & 1 & 2 & OV & 0.33 & $1-9$ \\
\hline 7 & Girl & 1 & 1 & 3 & $\mathrm{OVT}$ & 1.00 & $>30$ \\
\hline 8 & Boy & 1 & 1 & 3 & VTEt & 3.00 & $>30$ \\
\hline 9.5 & Girl & 6 & 2 & 1 & V & 0.17 & $10-30$ \\
\hline 10.5 & Boy & 8 & 2 & 1 & $C$ & 0.33 & $<1$ \\
\hline 10.5 & Girl & 3 & 2 & 1 & 0 & 0.25 & $<1$ \\
\hline 11.5 & Boy & 4 & 4 & 1 & C & 0.13 & $1-9$ \\
\hline 12.5 & Girl & 5 & 5 & 2 & V Ga & 0.20 & $>30$ \\
\hline 12.5 & Boy & 2 & 2 & 1 & C & 1.00 & $>30$ \\
\hline 12.5 & Girl & 9 & 8 & 3 & CVPh & 1.00 & $<1$ \\
\hline 13 & Boy & 1 & 0 & 2 & $V L$ & 0.22 & $<1$ \\
\hline 14.5 & Boy & 2 & 2 & 1 & 0 & 1.00 & $<1$ \\
\hline 14.5 & Boy & 4 & 3 & 1 & V & 0.50 & $1-9$ \\
\hline 15 & Boy & 5 & 4 & 2 & $C L$ & 1.00 & $10-30$ \\
\hline
\end{tabular}

falling into five domains: physical function (physical restrictions, energy/fatigue), cognitive function (attention/concentration, memory, language, other cognitive), emotional well-being (depression, anxiety, control/helplessness, self-esteem), social function (social interactions, social activities, stigma item) and behavior. The parental judgment of their child's poor functioning is reflected by the lower score in overall scale as well as in any of 16 sub-domains. The Polish translation was approved by the authors of the original scale and its high reliability and validity were demonstrated.

\section{Procedure}

During a visit in one of the specialist outpatient clinics, the child's parent/caregiver was asked to complete the QOLCE while the investigator was taking care of a child in a neighboring room. Prior to the interview, each child received an evaluation by a pediatric neurologist and neuropsychologist. As a part of the neurological evaluation each child underwent an EEG examination that was interpreted by a trained clinician. In addition, 23 out of 31 children underwent cranial computed tomography or magnetic resonance imaging. 


\section{Statistical analysis}

To test the main hypothesis, we conducted correlation analysis between lateralization and reduced QOL (overall QOL < 60; non-parametric Spearman's correlation). Two-sample t-tests for all sub-scales and domains were employed to describe the profile of impairments in the right- over the left-hemisphere group. Furthermore, leftand right-hemisphere groups were compared with regards to age, age of seizure onset, duration of disease, duration of medication, number and frequency of antiepileptic drugs (AED), number of hospitalizations per year, and average frequency of epileptiform discharges using two-sample t-tests. The level of significance was set according to $\mathrm{p}<0.05$ for all tests. All statistical analyses were performed with SPSS 14 software package (SPSS Inc., Chicago, IL).

\section{Results}

Confirming the main hypothesis, lateralization and reduced overall QOL correlated significantly (Spearman's rho $0.361, \mathrm{p}<0.046)$. Children with right-hemispheric epileptiform discharges exhibited a lower overall QOL. Table 2 shows the comparisons for all domains, subscales, and items of the QOLCE. T-tests revealed that in particular the social functioning domain, the anxiety subscale as well as the quality-of-life, stigma, and general health items were rated as more compromised in right-hemispheric children. There was no statistically significant difference between the groups in terms of age or any of the clinical measures (all p > 0.13 ) and the gender was distributed similarly (6 girls and 9 boys in the left-hemispheric, 6 girls and 10 boys in the right-hemispheric group).

\section{Discussion}

We have demonstrated for the first time that in children lateralized epileptiform discharges have a differential impact on QOL. However, in contrast to adults [13,24], the right-hemispheric foci more often led to lower QOL. In particular, the lateralization affected emotional and social domains, including anxiety, social activities and stigma.

The studies concerning lateralization of emotions are not conclusive so far: the right hemisphere was suggested to dominate in the processing of emotional information. However the left hemisphere was considered to be more involved in the processing of positive emotions (for a review, see [25]). The association of negative emotional outcome with right-hemispheric seizures in adults is often explained by the "valence" hypothesis that

Table 2 Mean score and standard deviation in QOLCE subscales for left- and right-hemispheric groups

\begin{tabular}{|c|c|c|c|c|}
\hline & Left hemisphere & Right hemisphere & $\mathrm{t}(29)$ & Significance \\
\hline Physical function & $57.9+11.4$ & $51.5+18.7$ & 1.156 & 0.128 \\
\hline Physical restrictions & $58.0 \pm 15.0$ & $47.2 \pm 24.2$ & 1.504 & 0.072 \\
\hline Energy/Fatigue & $57.8 \pm 12.0$ & $55.8 \pm 17.6$ & 0.368 & 0.358 \\
\hline Cognitive function & $58.6+19.8$ & $55.8+26.4$ & 0.337 & 0.369 \\
\hline Attention/Concentration & $66.6 \pm 23.7$ & $53.3 \pm 26.0$ & 1.483 & 0.074 \\
\hline Memory & $57.8 \pm 20.4$ & $58.1 \pm 24.3$ & -0.030 & 0.488 \\
\hline Language & $54.3 \pm 23.7$ & $59.0 \pm 28.0$ & -0.501 & 0.310 \\
\hline Other cognitive & $55.7 \pm 20.1$ & $52.8 \pm 31.8$ & 0.311 & 0.379 \\
\hline Emotional wellbeing & $68.7+10.9$ & $60.1+58.6$ & 1.520 & 0.069 \\
\hline Depression & $73.4 \pm 13.6$ & $65.0 \pm 17.8$ & 1.489 & 0.074 \\
\hline Anxiety & $71.8 \pm 19.9$ & $58.6 \pm 22.9$ & 1.726 & 0.048 \\
\hline Control/Helplessness & $61.7 \pm 20.8$ & $53.3 \pm 22.1$ & 1.088 & 0.143 \\
\hline Self-esteem & $67.8 \pm 13.2$ & $63.3 \pm 23.9$ & 0.652 & 0.260 \\
\hline Social function & $87.7+24.6$ & $67.6+31.4$ & 1.987 & 0.028 \\
\hline Social interactions & $90.1 \pm 33.9$ & $71.7 \pm 33.3$ & 1.526 & 0.069 \\
\hline Social activities & $79.2 \pm 16.1$ & $64.4 \pm 29.6$ & 1.734 & 0.047 \\
\hline Stigma item & $93.7 \pm 37.1$ & $66.7 \pm 34.9$ & 2.090 & 0.023 \\
\hline Behavior & $66.4 \pm 15.1$ & $58.9 \pm 16.9$ & 1.298 & 0.102 \\
\hline General-health item & $59.4 \pm 22.1$ & $43.3 \pm 27.5$ & 1.795 & 0.042 \\
\hline QOL item & $60.9 \pm 18.2$ & $38.3 \pm 22.9$ & 3.055 & 0.002 \\
\hline Overall QOL & $67.2 \pm 12.1$ & $56.9 \pm 19.7$ & 1.769 & 0.044 \\
\hline
\end{tabular}

Spearman's correlation analysis revealed lateralization effect. Significant differences for separate subscales between the groups, as revealed by two-sample t-test, are marked in bold. 
associates negative emotions with the right hemisphere and positive with the left hemisphere [13]. The seizures or functional lesion in the left hemisphere could lead to deficits in processing of positive emotions, effecting the accentuation of dysphoric mood, whereas lesions of the left hemisphere would lead to deficits in processing of positive emotions. Kirsch [26] proposed an alternative view, linking psychosocial disturbances in epilepsy with subtle cognitive deficits. Those subclinical deficits, such as disturbed perception or interpretation of emotional prosody, influence social interactions and may lead to significant deficits in social functioning later in life.

Childhood is a critical period for developing cognitive and social skills. Therefore a disease that starts early in life may disrupt the acquisition of social cognition skills [27]. The earlier such deficits start, the more severe the consequences might be, leading to disturbed 'social cognition', that is the ability to interpret and understand oneself, others and the social world. Right temporal seizures often lead to subtle cognitive deficits described by Kirsch [26], such as disturbed recognition of fear [28] or problems with recognizing and identifying famous faces [29]. This model is in accordance with results of Cohen et al. [17], who demonstrated that children with right temporal focus had worse performance in recognizing emotional prosody than healthy controls. Such deficit in prosody recognition could be the cause of increased anxiety and stigma perception and disruption of social activities, leading in consequence to a lower general QOL. In all the children in our study, the disease occurred at least one year prior to the study, but usually much earlier, allowing the potential deficits to evoke the long-lasting effects on psychosocial functioning.

Ott et al. [30] demonstrated that even $66 \%$ of children with epilepsy can meet the criteria of a DSM-IV psychiatric diagnosis, although only about $30 \%$ of them receives any psychiatric treatment. These comorbidities have an impact on the life of child and the entire family [31]. Consequently, childhood-onset epilepsy has a longterm adverse impact on health-related QOL, even in adults who are seizure free, off medications for many years [32]. Camfield et al. [7] demonstrated in the study of 504 children with epilepsy, that most of the clinical parameters of treatment and seizure control, including remission, did not predict children's social functioning. It was observed that mood is the strongest predictor of QOL in epilepsy as compared to neuropsychological measures such as psychomotor speed, verbal memory, language, and cognitive inhibition [33].

Since patient's QOL may be improved by managing psychiatric and neuropsychological issues, even more than by reducing seizure frequency, Salpekar \& Dunn [34] suggested, that the comprehensive treatment should take into account the etiology of psychiatric disturbances in epilepsy (e.g. pathophysiology of the brain, psychosocial stress of chronic epilepsy, medication side effects) and involve neurological, psychiatric as well as psychological modalities. Our results contribute to the understanding of developmental aspects of the link between brain pathophysiology and psychosocial functioning. Moreover, they create a basis for the development of more directed treatment strategies for young patients with unilateral seizures. Our study does not reveal the mechanism of the differences between leftand right-hemispheric groups. It points out, however, the importance of developmental aspects of the impact of seizures and allows for formulating directed hypotheses when planning future studies.

We found no statistically significant difference between the groups in their level of depression, which is often described in adults [11]. Since the questionnaires were filled by the parents of the children, not by the children themselves, one cannot exclude that parents did not notice the depressive features of their children. The possibility to miss signs of depression is the biggest disadvantage in the use of parental scales [35]. However, in a study by Mathiak et al. [18] there was also no higher depressiveness revealed in children with lateralized seizures, despite the use of self-report scales. Children in the left-hemispheric group attributed control over successful events in their lives to external sources, which is known to lead to depression later in life; the higher level of depressiveness found in adults with lefthemispheric focus may be a result of long-lasting, dysfunctional control attribution.

We decided not to assess the handedness of our subjects. While the relationship between handedness and hemispheric dominance appears to be strong in healthy right-handers (over 95\%), it is much harder to draw any conclusions in left-handed or ambidextrous subjects, over $60 \%$ of them are reported to have left hemispheric dominance (for a review, see [36]). Hemispheric dominance correlates to a much lower extent with handedness in epilepsy, both in right- and left-handers [37]. Consequently, we decided not to exclude left-handed subjects from our study. To control reliably for hemispheric dominance we would need to apply invasive (e.g. Wada testing) or technically demanding and expensive (fMRI) techniques that were out of scope of our study. If some of our subjects had a reversed hemispheric dominance pattern, this would result in decrease of a statistical power of our results. It limits, therefore, conclusions concerning negative findings.

Although children with anatomical malformations were excluded from the study, 8 subjects did not undergo neuroimaging. However our group encompassed children with benign rolandic epilepsy. These patients may not be referred to brain imaging by their 
neurologist. Based on the clinical information and EEG alone we expected no anatomical malformations in those subjects [38]. If some of them had had bilateral or contralateral lesions that were not detected in the neuroimaging or not diagnosed due to a lack of clinical signs, it would have resulted in a reduced power of the study, just like undetected contralateral foci. As a consequence the effect sizes may be underestimated.

A limitation of the present study is the size of this strictly selected group of children with unilateral foci and no anatomical malformations. The statistical power does not allow for an assessment of lobar localization, which could provide a better insight into the neurobiological background of the observed disturbance. However, other studies involving child populations, fulfilling strict criteria of unilateral seizures and no structural lesions, encompass usually comparable or smaller groups only ([17]: 23 children; [39]: 12 children; [40]: 27 children; [41]: 12 children). Combining additional diagnostic methods, such as magnetoencephalography (MEG), functional magnetic resonance imaging (fMRI) and intensive video-EEG monitoring, in future studies on focus lateralization might help overcome the problem of a small group size and give a better insight into the understanding of the underling pathologies.

\section{Conclusions}

Lateralization of epileptic focus can affect psychosocial functioning and lead to diminished QOL in children. Right-hemispheric foci may result in lower QOL, in particular in the domain of social functioning and emotions (anxiety). Focusing on the differences in QOL as a result of focus lateralization in development may have an important impact on treatment strategies and demands further studies. Taking into account developmental aspects in the course of epilepsy should facilitate understanding the complex influence of epileptic seizures on the brain structures. Childhood epilepsy can serve as a valuable neurobiological model to study the brain-behavior relationship.

\section{Acknowledgements}

The authors thank Dr. Dybała, Dr. Jeziorek, Dr. Świętochowska and Dr. Bielicka for their support in recruiting the subjects. We thank Dr. Mara Sittampalam for language corrections. This study was supported by the grant 2H01F04325, State Committee for Scientific Research in Poland and BST 144520, Faculty of Psychology, University of Warsaw, Poland. K.A. Mathiak was founded by JARA-Brain Project Realtime-fMRI, Exzellenzinitiative des Bundes und der Länder.

\section{Author details}

${ }^{1}$ Department of Child and Adolescent Psychiatry and Psychotherapy, RWTH Aachen University, Germany. ${ }^{2}$ Faculty of Psychology, University of Warsaw, Poland. ${ }^{3}$ Department of Psychiatry and Psychotherapy, RWTH Aachen University, Germany. ${ }^{4}$ INM-1, Forschungszentrum Jülich GmbH, Germany. ${ }^{5}$ Department of Child Psychiatry, Medical University of Warsaw, Poland. ${ }^{6}$ Clinic of Neurology of Children and Adolescents, Institute of Mother and Child, Warsaw, Poland.

\section{Authors' contributions}

KAM conceived and designed the study, acquired the funding, participated in and supervised collection of data, performed statistical analysis, and drafted the manuscript. M $Ł$ participated in collection of data and contributed to interpretation of results. KM participated in statistical analysis, contributed to interpretation of results and helped to draft the manuscript. KK participated in statistical analysis. TW participated in the design of the study. ES coordinated acquisition of clinical data and consulted clinical criteria of including patients. PO participated in the design and coordination of the study. All authors read and approved the final manuscript.

\section{Competing interests}

The authors declare that they have no competing interests.

Received: 16 December 2009 Accepted: 17 August 2010

Published: 17 August 2010

\section{References}

1. Devinsky O, Cramer JA: Quality of Life in Epilepsy. Epilepsia 1993, 34(Suppl 4):1-3.

2. The WHOQOL Group: The World Health Organization Quality of Life Assessment (WHOQOL): position paper from World Health Organization. Soc Sci Med 1995, 41:1403-1409.

3. Baker GA: Quality of life and epilepsy: the Liverpool experience. Clin Ther 1998, 20(Suppl A):2-12

4. Ronen GM, Streiner DL, Rosenbaum P: Health-related quality of life in childhood epilepsy: moving beyond 'seizure control with minimal adverse effects'. Health Qual Life Outcomes 2003, 28:1-36.

5. Baker GA, Hargis E, Mo-Song Hsih M, Mounfield H, Arzimanoglou A, Glauser T, Pellock J, Lund S: Perceived impact of epilepsy in teenagers and young adults: An international survey. Epilepsy Behav 2008, 12:395-401.

6. Vickrey BG, Hays RD, Rausch R, Sutherling WW, Engel J Jr, Brook RH: Quality of life of epilepsy surgery patients as compared with outpatients with hypertension, diabetes, heart disease, and/or depressive symptoms. Epilepsia 1994, 35:597-607.

7. Camfield C, Camfield P, Smith B, Gordon K, Dooley J: Biologic factors as predictors of social outcome of epilepsy in intelectually normal children: A population-based study. J Pediatr 1993, 122:869-873.

8. Hermann BP: Developing a Model of Quality of Life in Epilepsy: The Contribution of Neuropsychology. Epilepsia 1993, 34(Suppl 4):S14-S21.

9. Devinsky O: Right cerebral hemisphere dominance for a sense of corporeal and emotional self. Epilepsy Behav 2000, 1:60-73.

10. Sackeim HA, Greenberg MS, Weiman AL, Gur RC, Hungerbuhler JP, Geschwind N: Hemispheric asymmetry in the expression of positive and negative emotions neurologic evidence. Arch Neurol 1982, 39:210-218.

11. Helmstaedter C, Sonntag-Dillender M, Hoppe C, Elger CE: Depressed mood and memory impairment in temporal lobe epilepsy as a function of focus lateralization and localization. Epilepsy Behav 2004, 5:696-701.

12. Piazzini A, Canevini MP, Maggiori G, Canger R: Depression and Anxiety in Patients with Epilepsy. Epilepsy Behav 2001, 2:481-489.

13. Andelman F, Fried I, Nuefeld MY: Quality of life self-assessment as a function of lateralization of lesion in candidates for epilepsy surgery. Epilepsia 2001, 42:549-555.

14. Grabowska A, Herman A, Nowicka A, Szatkowska I, Szelag E: Individual differences in the functional asymmetry of the human brain. Acta Neurobiol Exp 1994, 54:155-162.

15. Upton D, Thompson PJ: Neuropsychological test performance in frontallobe epilepsy: the influence of aetiology, seizure type, seizure frequency and duration of disorder. Seizure 1997, 6:443-447.

16. Austin JK, Caplan R: Behavioral and psychiatric comorbidities in pediatric epilepsy: toward an integrative model. Epilepsia 2007, 48:1639-1651.

17. Cohen M, Prather A, Town P, Hynd G: Neurodevelopmental differences in emotional prosody in normal children and children with left and right temporal lobe epilepsy. Brain Lang 1990, 38:122-134

18. Mathiak KA, Mathiak K, Wolańczyk T, Ostaszewski P: Psychosocial impairments in children with epilepsy depend on the side of the focus. Epilepsy Behav 2009, 16:603-608.

19. Vickrey $B G$, Hays RD, Brook RH, Rausch R: Reliability and validity of the Katz Adjustment Scales in an epilepsy sample. Qual Life Res 1992, 1(1):63-72 
20. Sabaz M, Cairns DR, Lawson JA, Nheu N, Bleasel AF, Bye AM: Validation of a new quality of life measure for children with epilepsy. Epilepsia 2000, 41:765-774.

21. Sabaz M, Lawson JA, Cairns DR, Duchowny MS, Resnick TJ, Dean PM: The impact of epilepsy surgery on quality of life in children. Neurology 2006 66:557-561.

22. Mathiak KA, Karzel K, Mathiak K, Ostaszewski P, Łuba M, Wolańczyk T: Polish adaptation and validation of the Health-Related Quality of Life in Childhood Epilepsy Questionnaire. Neurol Neurochir Pol 2007, 41:203-214.

23. Sabaz M, Lawson JA, Cairns DR, Duchowny MS, Resnick TJ, Dean PM, Bye AM: Validation of the quality of life in childhood epilepsy questionnaire in American epilepsy patients. Epilepsy Behav 2003, 4:680-691.

24. Lambert MV, Robertson MM: Depression in epilepsy: etiology, phenomenology and treatment. Epilepsia 1999, 40(Suppl 10):21-47.

25. Silberman EK, Weingartner $\mathrm{H}$ : Hemispheric Lateralization of Functions Related to Emotion. Brain Cogn 1986, 5:322-353.

26. Kirsch HE: Social cognition and epilepsy surgery. Epilepsy Behav 2006, 8:71-80.

27. Austin JK, Smith MS, Risinger MW, McNelis AM, Childhood epilepsy and asthma: comparison of quality of life. Epilepsia 1994, 35:608-15.

28. Meletti S, Benuzzi F, Rubboli G, Cantalupo G, Stanzani MM, Nichelli P: Impaired facial emotion recognition in early-onset right mesial temporal lobe epilepsy. Neurology 2003, 60:426-431.

29. Seidenberg M, Berent S: Childhood epilepsy and the role of psychology. The American Psychologist 1992, 47:1130-1133.

30. Ott D, Siddarth P, Gurbani S, Koh S, Tournay A, Shields WD, Caplan R: Behavioral disorders in pediatric epilepsy: unmet psychiatric need. Epilepsia 2003, 44:591-597.

31. Sillanpää $M$, Cress JH: The psychosocial impact of epilepsy in childhood. Epilepsy Behav 2009, 15(Suppl 1):5-10.

32. Sillanpää M, Haataja L, Shinnar S: Perceived impact of childhood-onset epilepsy on quality of life as an adult. Epilepsia 2004, 45:971-977.

33. Perrine K, Hermann BP, Meador K, Vickerey BG, Cramer JA, Hays RD, Devinsky O: The relationship of neuropsychological functioning to quality of life in epilepsy. Arch Neurol 1995, 52:997-1003.

34. Salpekar JA, Dunn DW: Consequences of Pediatric Epilepsy. Semin Pediatr Neurol 2007, 14:181-188.

35. Leon GR, Kendall PC, Garber J: Depression in children: parent, teacher, and child perspectives. J Abnorm Child Psychol 1980, 8:221-235

36. Hellige JB: Hemispheric asymmetry. Annu Rev Psychol 1990, 41:55-80.

37. L Medina S, Bernal B, Ruiz J: Role of functional MR in determining language dominance in epilepsy and nonepilepsy populations: a Bayesian analysis. Radiology 2007, 242:94-100

38. Gaillard WD, Chiron C, Cross JH, Harvey AS, Kuzniecky R, Vezina LG, ILAE, Committee for Neuroimaging, Subcommittee for Pediatric Neuroimaging: Guidelines for imaging infants and children with recent-onset epilepsy. Epilepsia 2009, 50:2147-2153.

39. Metz-Lutz MN, Kleitz C, de Saint Martin A, Massa R, Hirsch E, Marescaux C: Cognitive development in benign focal epilepsies of childhood. Dev Neurosci 1999, 21:182-90.

40. Kolk A, Beilmann A, Tomberg T, Napa A, Talvik T: Neurocognitive development of children with congenital unilateral brain lesion and epilepsy. Brain Dev 2001, 23:88-96.

41. Bedoin N, Herbillon V, Lamoury I, Arthaud-Garde P, Ostrowsky K, De Bellescize J, Kéo Kosal P, Damon G, Rousselle Ch: Hemispheric lateralization of cognitive functions in children with centrotemporal spikes. Epilepsy Behav 2006, 9:268-74.

\section{Pre-publication history}

The pre-publication history for this paper can be accessed here: http://www.biomedcentral.com/1471-2377/10/69/prepub

\section{doi:10.1186/1471-2377-10-69}

Cite this article as: Mathiak et al: Quality of life in childhood epilepsy with lateralized epileptogenic foci. BMC Neurology 2010 10:69. 